\title{
In vitro and in vivo study of a nanoliposomal cisplatin as a radiosensitizer
}

\author{
This article was published in the following Dove Press journal: \\ International Journal of Nanomedicine \\ 2I February 20II \\ Number of times this article has been viewed
}

\author{
Xiaomeng Zhang ${ }^{\text {* }}$ \\ Huanjun Yang ${ }^{*}$ \\ $\mathrm{Ke} \mathrm{Gu}$ \\ Jian Chen ${ }^{2}$ \\ Mengjie Rui ${ }^{2}$ \\ Guo-Liang Jiang' \\ 'Departments of Radiation Oncology, \\ Fudan University Shanghai Cancer \\ Center; Department of Oncology, \\ Shanghai Medical College,Fudan \\ University,Shanghai, People's Republic \\ of China; ${ }^{2}$ School of Pharmacy, \\ Shanghai Jiao Tong University, \\ Shanghai, People's Republic of China; \\ *Xiaomeng Zhang and Huanjun Yang \\ share the first authorship
}

Objective: To investigate the in vitro and in vivo radiosensitization effect of an institutionally designed nanoliposome encapsulated cisplatin (NLE-CDDP).

Materials and methods: NLE-CDDP was developed by our institute. In vitro radiosensitization of NLE-CDDP was evaluated by colony forming assay in A549 cells. In vivo radiosensitization was studied with tumor growth delay (TGD) in Lewis lung carcinoma. The radiosensitization for normal tissue was investigated by jejunal crypt survival. The radiosensitization studies were carried out with a $72 \mathrm{~h}$ interval between drug administration and irradiation. The mice were treated with $6 \mathrm{mg} / \mathrm{kg}$ of NLE-CDDP or CDDP followed by single doses of 2 Gy, 6 Gy, 16 Gy, and 28 Gy. Sensitization enhancement ratio (SER) was calculated by $\mathrm{D}_{0} \mathrm{~s}$ of cell survival curves for A549 cells, doses needed to yield TGD of 20 days in Lewis lung carcinoma, or $\mathrm{D}_{0} \mathrm{~s}$ of survival curves in crypt cells in radiation alone and radiation plus drug groups.

Results: Our NLE-CDDP could inhibit A549 cells in vitro with half maximal inhibitory concentration of $1.12 \mu \mathrm{g} / \mathrm{mL}$, and its toxicity was 2.35 times that observed in CDDP. For in vitro studies of A549 cells, SERs of NLE-CDDP and CDDP were 1.40 and 1.14, respectively, when combined with irradiation. For in vivo studies of Lewis lung carcinoma, the strongest radiosensitization was found in the $72 \mathrm{~h}$ interval between NLE-CDDP and irradiation. When given $72 \mathrm{~h}$ prior to irradiation, NLE-CDDP yielded higher radiosensitization than CDDP (SER of 4.92 vs 3.21) and slightly increased injury in jejunal crypt cells (SER of 1.15 vs 1.19). Therefore, NLE-CDDP resulted in a higher TGF than did CDDP (4.28 vs 2.70) when SERs were compared between experiments in vivo and in jejunal crypt cell studies.

Conclusions: Our NLE-CDDP was demonstrated to have radiosensitization with TGF of 4.28 when administrated $72 \mathrm{~h}$ prior to irradiation.

Keywords: cisplatin, liposome, nanoparticle, radiation, radiosensitization

\section{Introduction}

Cisplatin (CDDP) is a highly effective antitumor agent and is applied widely in the treatment of various cancers. Furthermore, CDDP is a commonly used radiosensitizer. However, after intravenous injection, the majority of CDDP is bound with plasma albumin to form an essentially irreversible complex with limited antitumor activity. In addition, the renal toxicity of CDDP limits its use. Therefore, it is important to study how to avoid or reduce plasma albumin binding of CDDP and improve its effective bioavailability. ${ }^{1-3}$

Liposome technologies, especially the long-circulating nanoliposome technologies, provide effective means to solve those problems. ${ }^{4,5}$ Liposomal CDDP can isolate agents from plasma albumin, and pegylated liposome is able to prolong the circulation time
Correspondence: Guo-Liang Jiang Departments of Radiation Oncology, Fudan University Shanghai Cancer Center; Department of Oncology, Shanghai Medical College,Fudan University,Shanghai 200032, People's Republic of China Tel +862164175590 Fax +862164439052 Email jianggl47@hotmail.com 
of free CDDP in blood. Nanoliposome can penetrate the interendothelial cell gap of nascent tumor capillaries much easier than of normal tissues, then deposit in the tumor. Those natural characteristics can increase CDDP concentration in the tumor while reducing CDDP concentration in normal tissues, thereby decreasing its toxicity. ${ }^{6-8}$ In this study, we investigated the radiosensitization effect in vitro and in vivo of a newly developed agent, nanoliposomal encapsulated CDDP (NLE-CDDP).

\section{Materials and methods Preparation of NLE-CDDP}

NLE-CDDP was produced in the School of Pharmacy, Shanghai Jiao Tong University, Shanghai, People's Republic of China, by the reverse-phase evaporation method, which was originally introduced by Szoka and Papahadjopoulos. ${ }^{9}$ The lipid agents included hydrogenated soybean phosphatidylcholine (HSPC, NOF corporation, Tokyo, Japan), cholesterol (Sigma-Aldrich, St Louis, MO), and 2000PEG-1, 2-distearoyl-sn-glycero-3-phosphoethanolamine (2000PEG-DSPE, NOF Corporation). CDDP was packed in central aqueous core of the liposomal nanoparticles at a ratio of 1:5 (CDDP vs lipid molar). The final NLE-CDDP consisted of small nanoliposomes of an average size of approximately $100 \mathrm{~nm}$ with CDDP concentration of $1 \mathrm{mg} / \mathrm{mL}$. The formulation was stable at room temperature. The CDDP remained at a concentration of $93 \%$ of the initial status even 1 month after preparation. In this study, NLECDDP was prepared immediately prior to use. CDDP was taken as the control, which was obtained from Shandong Qilu Pharmaceutical Company (Lot number 060924).

\section{Cell culture}

A549 human lung adenocarcinoma cell line was used in this study, incubated in RPMI medium $1640\left(\mathrm{GIBCO}^{\circledR}\right.$, Invitrogen, Gaithersburg, MD) with $10 \%$ fetal bovine serum (Biochrom, Berlin, Germany) at $37^{\circ} \mathrm{C}$ in a humidified atmosphere of $5 \% \mathrm{CO}_{2}$. Logarithmic growth cells were harvested for experiments.

\section{Methylthiazoltetrazolium (MTT) assay}

A549 cells were seeded in 96-well plates with a density of 3000 cells $/ 90 \mu \mathrm{L}$ per well and incubated overnight. NLE-CDDP and CDDP solutions were prepared with RPMI 1640 medium without serum and added to the cells, $10 \mu \mathrm{L}$ per well, with final drug concentrations of $0.1 \mu \mathrm{g} / \mathrm{mL}, 0.3 \mu \mathrm{g} / \mathrm{mL}$, $0.5 \mu \mathrm{g} / \mathrm{mL}, 1 \mu \mathrm{g} / \mathrm{mL}, 3 \mu \mathrm{g} / \mathrm{mL}, 5 \mu \mathrm{g} / \mathrm{mL}$, and $10 \mu \mathrm{g} / \mathrm{mL}$ in medium, respectively. Eight replicates were performed for each drug dose. Every well plate had two controls: saline and culture medium. After cells were incubated for 48 h, $20 \mu \mathrm{L}$ 3-(4, 5-dimethylthiazol-2-y1)-2, 5-diphenytetrazolium bromide was added in each well and remained for $4 \mathrm{~h}$. Then $100 \mu \mathrm{L}$ solubilization solution was added into the medium. The absorbance of the colored solution was quantified by a spectrophotometer at $570 \mathrm{~nm}$ with a reference wavelength of $630 \mathrm{~nm}$. Inhibition of cell viability was calculated by the formula: $\left[1-\left(\mathrm{OD}_{\text {test }}-\mathrm{OD}_{\text {blank }}\right) /\right.$ $\left(\mathrm{OD}_{\text {control }}-\mathrm{OD}_{\text {blank }}\right)$ ], and half maximal inhibitory concentration (IC50) was derived from GraphPad Prism. Free liposomes were also tested for cell inhibition by MTT.

\section{Colony forming assay}

A549 cells were incubated in $25 \mathrm{~cm}^{2}$ flasks overnight then exposed to NLE-CDDP or CDDP at a concentration of $0.5 \mu \mathrm{g} / \mathrm{mL}$, or irradiated. Drugs were administrated $24 \mathrm{~h}$ before irradiation. Cells were irradiated using a $6 \mathrm{MV}$ X-ray linear accelerator at a dose rate of $1.24 \mathrm{~Gy} / \mathrm{min}$. Two hours after irradiation, cells were trypsinized, counted, and seeded in $6 \mathrm{~cm}$ dishes with $5 \mathrm{~mL}$ medium at appropriate concentrations. There were three dishes at each dose. The cells were incubated for 10 days and then stained with crystal violet. Colonies with more than 50 cells were counted. Cell survival curve was estimated by a multitarget single-hit model $\left(S=1-\left(1-e^{-D / D 0}\right)^{N}\right)$ (L-Q) and then $\mathrm{D}_{0}$ was calculated.

\section{In vivo tumor inhibition test}

The in vivo study was approved by the Animal Study Committee and was conducted in accordance with Fudan University guidelines and regulations on the use and care of laboratory animals. Lewis lung carcinoma was implanted in the right flank of C57BL/6N male mice (6 weeks old and weight $17 \pm 1.8 \mathrm{~g}$ ). Mice were purchased from the Experimental Animal Center, Chinese Academy of Sciences, Shanghai, and maintained in specific pathogen-free rooms. The in vivo study was started 5 to 7 days after implantation when flank tumors grew to diameters of $8-10 \mathrm{~mm}$.

Lewis lung carcinoma-bearing mice were randomly assigned to one of the following groups: CDDP alone, NLE-CDDP alone, irradiation alone, CDDP followed by irradiation, NLE-CDDP followed by irradiation, and control with no treatments. Drugs were injected through the mouse tail vein at a dose of $6 \mathrm{mg} / \mathrm{kg}$. In order to investigate whether NLE-CDDP can enhance the radiation effect even after a long time, we designed experiments with different intervals between drug administration and irradiation. A single dose of 6 Gy irradiation was delivered $1 \mathrm{~h}, 24 \mathrm{~h}$, and $72 \mathrm{~h}$ after drug delivery. To further investigate the interval for the $72 \mathrm{~h}$ 
group, single doses of 0 Gy, 2 Gy, 6 Gy, 16 Gy, and 28 Gy were given in the irradiation-alone and combination of irradiation and drugs groups.

Tumors were measured three times a week until the death of the mice or 60 days after treatment. Tumor volume was calculated by $V=\left(a^{*} b^{2}\right) / 2$, where $a$ was the maximum diameter and $b$ was the orthogonal diameter. However, a relative tumor volume was used, which was a ratio of tumor volume after treatment versus the pretreatment volume. Tumor growth curves were constructed according to the Gompertz model with the equation $y=V_{0}^{*} \exp \left(k^{*}\left(1-\exp \left(-a^{*} X\right)\right)\right)$, where $V_{0}$ was the original tumor volume; $k$ and $a$, coefficient; $X$, irradiation dose, and $y$ tumor growth delay (TGD) time. TGD in days was defined as the difference between $\mathrm{T}_{5 \mathrm{~V} 0}$ of treated tumors compared with untreated tumors. $\mathrm{T}_{5 \mathrm{~V} 0}$ was the days needed for tumor growth from the original size to five times its original volume.

\section{Enhancement of irradiation injury in intestinal epithelium}

A total of 75 mice was divided into three groups: radiation alone, CDDP and radiation, and NLE-CDDP and radiation. Each group included five subgroups that received total abdomen irradiation of $0 \mathrm{~Gy}, 8 \mathrm{~Gy}, 10 \mathrm{~Gy}, 12 \mathrm{~Gy}$, and $14 \mathrm{~Gy}$, respectively. Irradiation was carried out $72 \mathrm{~h}$ after NLECDDP or CDDP injection. The mice were sacrificed 3.5 days after irradiation. Then a segment of jejunum was removed and its transverse slices were sectioned for hematoxylin and eosin staining. The number of crypts per circumference was counted under microscope, and crypt survival was then calculated. The jejunal crypt survival curves were determined by linear-quadratic model (L-Q) with the equation $y=\exp \left(-\alpha^{*} D-\beta^{*} D^{2}\right)$, where $\alpha$ and $\beta$ were coefficient; $D$, irradiation dose; and $y$, survival faction. Sensitization enhancement ratio (SER) was the ratio of $\beta$ value of the combination to radiation alone group.

\section{Statistics}

Comparisons of mean value were performed by Student's $t$-test and one-way analysis of variance (ANOVA). Comparisons of tumor growth were performed using repeated-measures ANOVA. The impact of irradiation and drugs on tumor growth was analyzed by the general linear model, and the differences among groups were compared by the Games-Howell method. The significance level was set at $P<0.05$.

For A549 cells, SER was defined as the ratio of $\mathrm{D}_{0}$ in radiation alone over $\mathrm{D}_{0}$ in the drugs and radiation groups.
The biological endpoint was defined as a TGD of 20 days for in vivo Lewis lung carcinoma, and SER was the ratio of radiation dose needed to reach the endpoint in the irradiation alone versus irradiation dose in combination groups. SER was the ratio of $\mathrm{D}_{0}$ in the radiation alone group over $\mathrm{D}_{0}$ in the drugs and radiation group for jejunal crypt.

\section{Results \\ NLE-CDDP and CDDP cytotoxicity in vitro}

No toxicity was detected in A549 cell line for free liposomes, whereas the survivals trend increased compared with that in the control group. Both NLE-CDDP and CDDP demonstrated inhibitions of A549 cells (Figure 1). IC50 of NLE-CDDP and CDDP was $1.12 \mu \mathrm{g} / \mathrm{mL}$ and $2.63 \mu \mathrm{g} / \mathrm{mL}$, respectively. NLE-CDDP was more toxic than CDDP when drug concentrations were $\leq 3 \mu \mathrm{g} / \mathrm{mL}(P<0.05)$, whereas when the concentrations increased to $5 \mu \mathrm{g} / \mathrm{mL}$ and $10 \mu \mathrm{g} / \mathrm{mL}$, both drugs showed similar inhibition.

\section{In vitro colony forming assay}

Cell survival curves are illustrated in Figure 2. $\mathrm{D}_{0} \mathrm{~s}$ was $1.406 \mathrm{~Gy}, 1.235 \mathrm{~Gy}$, and $1.005 \mathrm{~Gy}$, respectively for the irradiation-alone, CDDP plus irradiation, and NLE-CDDP plus irradiation groups. SERs of CDDP and NLE-CDDP were 1.14 and 1.40 , respectively $(P=0.043)$.

\section{In vivo drug radiosensitization when combined with 6 Gy at different intervals}

Tumor growth curves are shown in Figure 3 and TGD in Table 1. Irradiation of 6 Gy alone yielded a TGD of 1.83 days, which was significantly different from the control $(P<0.05)$

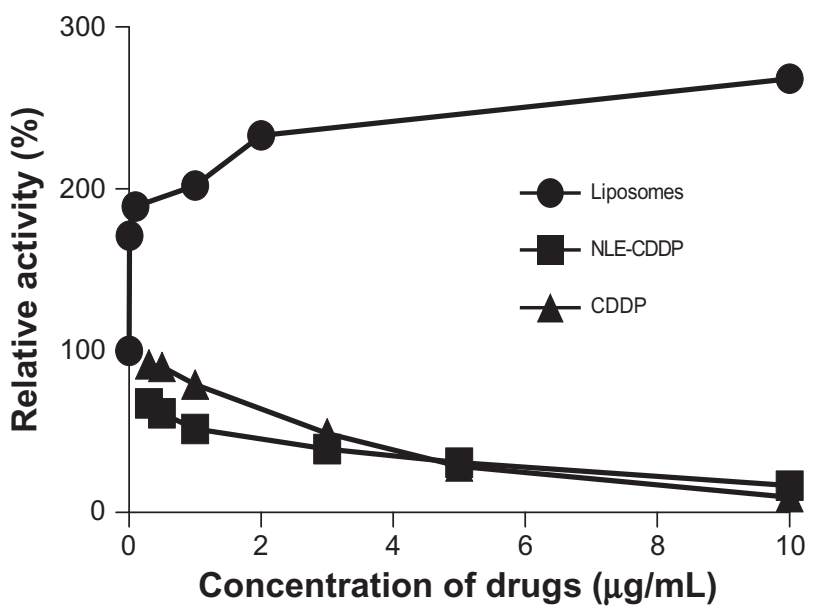

Figure I Effect of free liposomes, NLE-CDDP, and CDDP on A549 cells. Abbreviations: CDDP, cisplatin; NLE-CDDP, nanoliposome encapsulated cisplatin. 


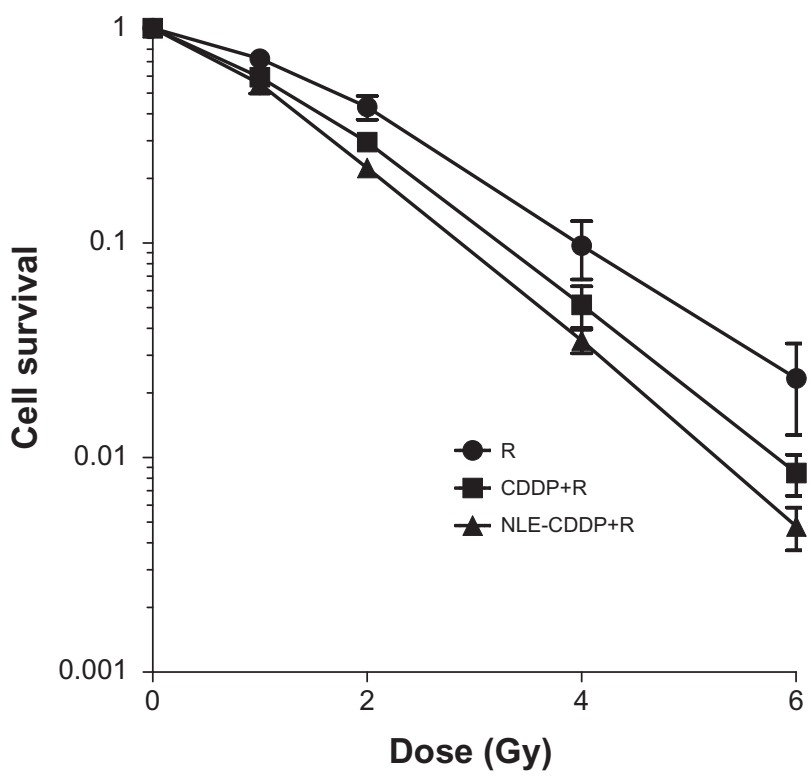

Figure 2 Cell survival curves after treatments with radiation alone $(R)$ or combined with $0.5 \mu \mathrm{g} / \mathrm{mL} \mathrm{NLE-CDDP} \mathrm{(NLE-CDDP} \mathrm{+} \mathrm{R)} \mathrm{or} \mathrm{CDDP}(C D D P+R)(P=0.00$ for CDDP vs $R ; P=0.00$ for NLE-CDDP vs $R ; P=0.043$ for CDDP vs NLE-CDDP).

Abbreviations: CDDP, cisplatin; NLE-CDDP, nanoliposome encapsulated cisplatin.

NLE-CDDP alone resulted in a longer TGD of 11.95 days rather than 3.27 days in CDDP. For combination groups, NLE-CDDP produced significantly longer TGD than CDDP did in $1 \mathrm{~h}, 24 \mathrm{~h}$, and $72 \mathrm{~h}$ interval groups, with $P$ values of $0.000,0.001$, and 0.0003 , respectively. Moreover, longer drug treatment time produced longer TGD, but statistical significance was found only in the NLE-CDDP group between the $1 \mathrm{~h}$ and $72 \mathrm{~h}$ treatment groups $(P=0.007)$. Variance analysis was performed and the impact of different intervals on TGD was statistically significant, with $F$ of 6.610 and $P$ value of 0.000 .

\section{In vivo drug radiosensitization when combined with irradiation}

\section{at $72 \mathrm{~h}$ interval}

In this experiment the interval was $72 \mathrm{~h}$ when drugs and irradiation were combined. The tumor growth curves are shown in Figure 4 and TGD in Table 2. With an increase in irradiation dose, tumor growth became slow and TGD was gradually extended in the irradiation-alone, NLE-CDDP, and CDDP groups. Drug plus irradiation prolonged TGD but was more pronounced for NLE-CDDP and irradiation $(P=0.002)$.

Dose response curves were generated for the irradiationalone, NLE-CDDP plus irradiation, and CDDP plus irradiation with a $72 \mathrm{~h}$ interval in combination groups (Figure 5) from data in Table 2. In Figure 5 the curves are nearly parallel when
A
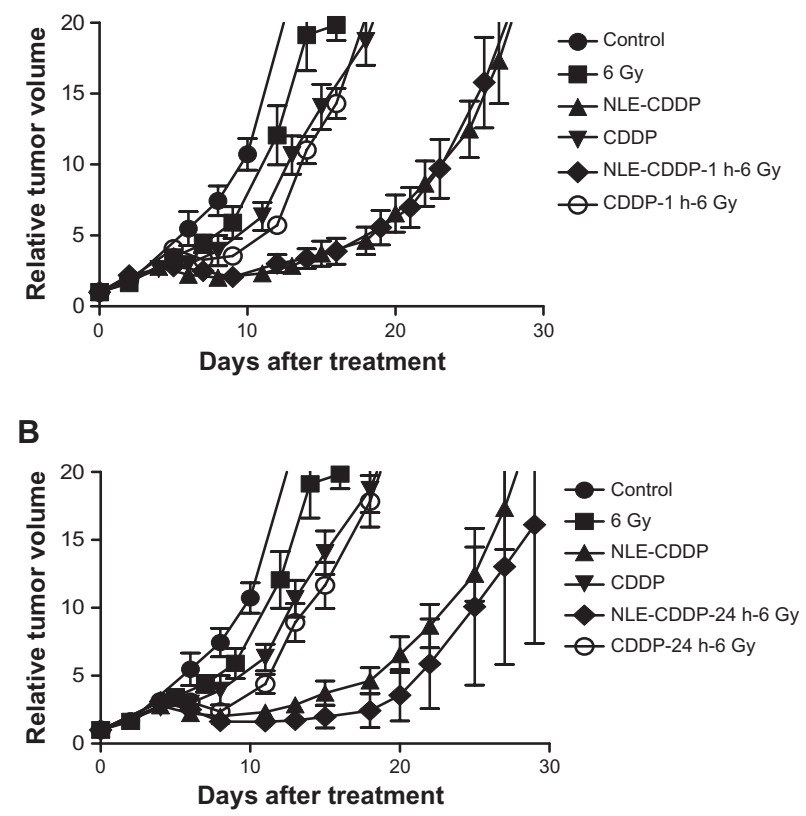

C

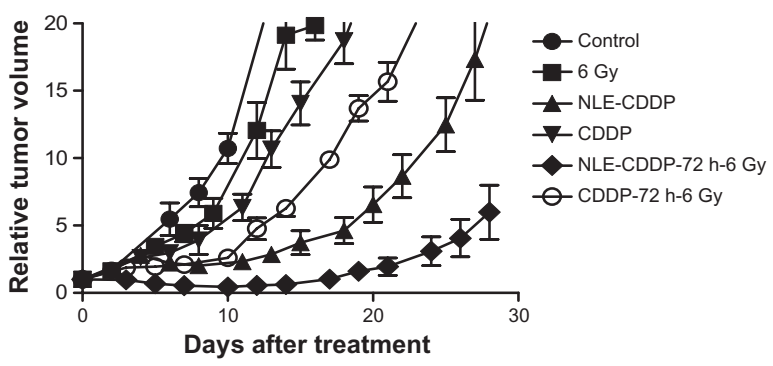

Figure 3 Tumor growth curves after irradiation of $6 \mathrm{~Gy}, 6 \mathrm{mg} / \mathrm{kg}$ of NLE-CDDP or CDDP or combinations of 6 Gy and NLE-CDDP/CDDP. A) Tumors treated with 6 Gy, NLE-CDDP, CDDP, or combinations of 6 Gy and NLE-CDDP/CDDP with I h interval between drugs and irradiation $(P=0.000)$. B) Tumors treated with $6 \mathrm{~Gy}$, NLE-CDDP, CDDP, or combinations of 6 Gy and NLE-CDDP/CDDP with $24 \mathrm{~h}$ interval between drugs and irradiation $(P=0.000)$. $(P=0.001)$. C) Tumors treated with $6 \mathrm{~Gy}$, NLE-CDDP, CDDP, or combinations of $6 \mathrm{~Gy}$ and NLE-CDDP/CDDP with $72 \mathrm{~h}$ interval between drugs and irradiation $(P=0.000)$. $(P=0.0003)$. (Relative tumor volume: a ratio of tumor volume after treatment versus the pretreatment volume.) Abbreviations: CDDP, cisplatin; NLE-CDDP, nanoliposome encapsulated cisplatin.

Table I Tumor growth delay in Lewis lung carcinoma after NLECDDP plus 6 Gy or CDDP plus 6 Gy with different intervals

\begin{tabular}{lll}
\hline Group & $\mathbf{T}_{\text {5v }}$ (days) & TGD (days) \\
\hline Control & 6.12 & 0 \\
6 Gy & 7.95 & 1.83 \\
NLE-CDDP & 18.07 & 11.95 \\
CDDP & 9.39 & 3.27 \\
NLE-CDDP-I h-6 Gy & 18.33 & 12.21 \\
NLE-CDDP-24 h-6 Gy & 19.16 & 13.04 \\
NLE-CDDP-72 h-6 Gy & 26.97 & 20.85 \\
CDDP-I h-6 Gy & 10.85 & 4.73 \\
CDDP-24 h-6 Gy & 11.11 & 4.99 \\
CDDP-72 h-6 Gy & 12.77 & 6.65 \\
\hline
\end{tabular}

Abbreviations: CDDP, cisplatin; NLE-CDDP, nanoliposome encapsulated cisplatin; $T G D$, tumor growth delay; $T_{5 v 0}$, the days needed for tumor growth from the original size to five times the original volume. 
A
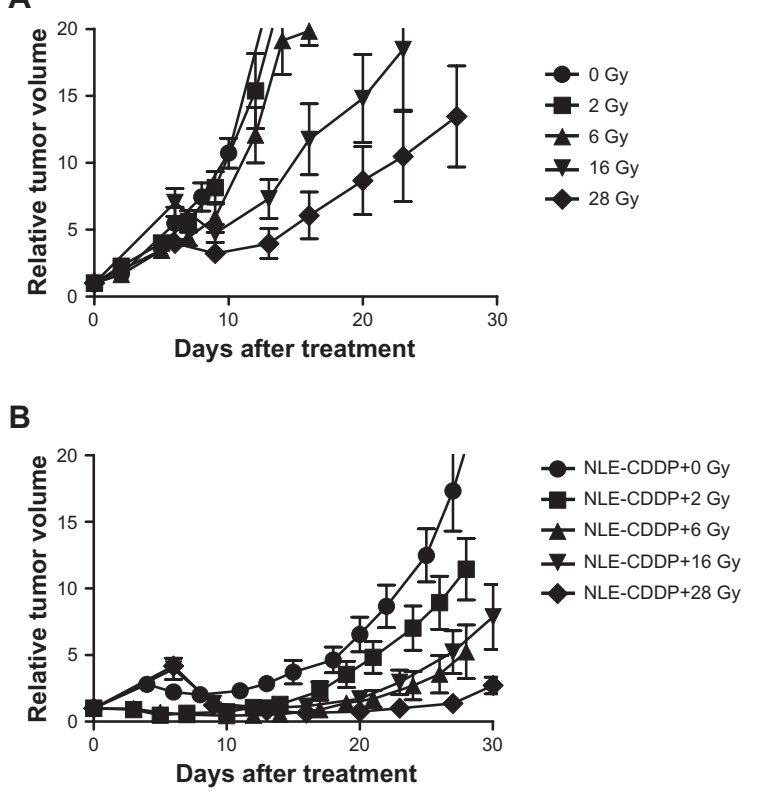

C

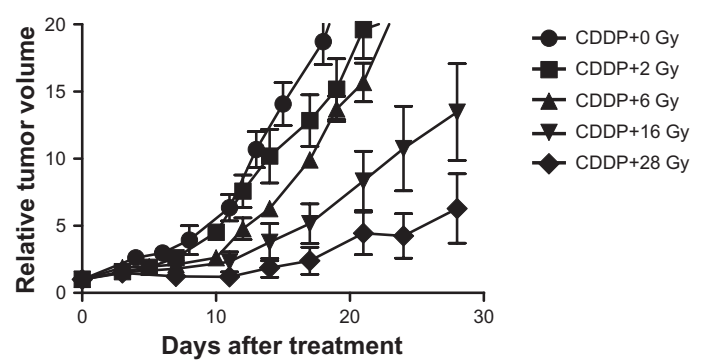

Figure 4 Tumor growth curves treated with a single dose of irradiation, NLECDDP plus irradiation, or CDDP plus irradiation with $72 \mathrm{~h}$ interval. A) Tumor treated with single doses of irradiation. B) Tumor treated with $6 \mathrm{mg} / \mathrm{kg}$ of NLECDDP followed by irradiation. C) Tumor treated with $6 \mathrm{mg} / \mathrm{kg}$ of CDDP followed by irradiation.

Abbreviations: CDDP, cis-platinum diammine dichloride; NLE-CDDP, nanoliposome encapsulated cisplatin.

TGDs were between 15 and 25 days. Thus, TGD of 20 days was taken as a biological endpoint. To achieve a TGD of 20 days, 38.1 Gy, 24.83 Gy, and 7.74 Gy were needed, respectively, for the irradiation-alone, CDDP plus irradiation, and NLE-CDDP plus irradiation groups. Therefore, when drugs were administrated $72 \mathrm{~h}$ before radiation, SER was $3.21(24.83 / 7.74)$ for CDDP and 4.92 (38.1/7.74) for NLE-CDDP.

Table 2 Tumor growth delay (days) in Lewis lung carcinoma after NLE-CDDP plus irradiation, or CDDP plus irradiation with $72 \mathrm{~h}$ interval

\begin{tabular}{llllll}
\hline Group & 0 Gy & 2 Gy & 6 Gy & 16 Gy & 28 Gy \\
\hline Irradiation-alone & 0 & 0.4 & 1.83 & 1.99 & 7.48 \\
NLE-CDDP + irradiation & 11.95 & 15.29 & 20.85 & 19.54 & 56.88 \\
CDDP + irradiation & 3.27 & 3.96 & 6.65 & 13.38 & 21.98 \\
\hline
\end{tabular}

Abbreviations: CDDP, cisplatin; NLE-CDDP, nanoliposome encapsulated cisplatin.

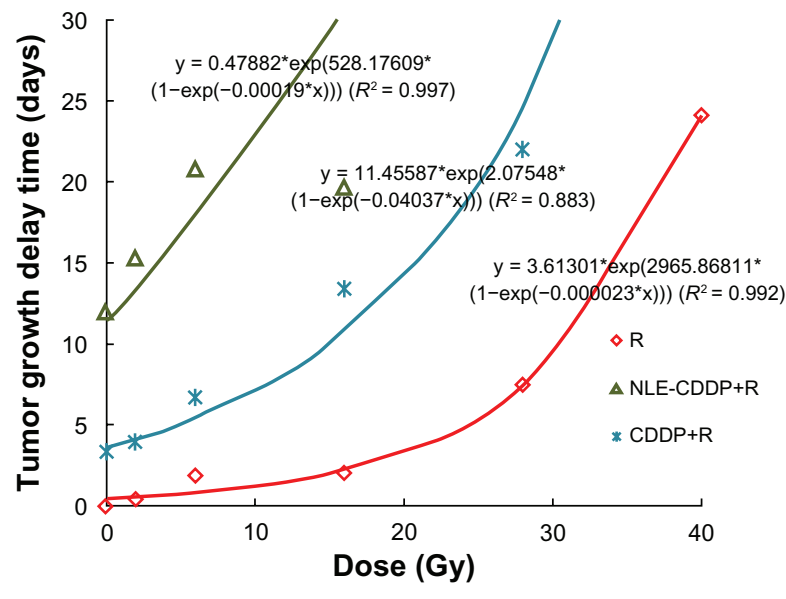

Figure 5 Dose-response curves from irradiation combined with NLE-CDDP or CDDP administered $72 \mathrm{~h}$ prior to irradiation in Lewis lung carcinoma. Abbreviations: CDDP, cis-platinum diammine dichloride; NLE-CDDP, nanoliposome encapsulated cisplatin; $R$, radiation alone.

\section{Drug radiosensitization for jejunum crypt cells}

Mice were sacrificed 3.5 days after treatment in order to count jejunum crypts. The number of survived jejunal crypts and survivals for each group are illustrated in Figure 6. When drugs were injected intravenously $72 \mathrm{~h}$ before irradiation, SERs were 1.15 for NLE-CDDP and 1.19 for CDDP based on the regression curves and L-Q model.

\section{Therapeutic gain factor (TGF)}

When drugs were used $72 \mathrm{~h}$ before irradiation, TGF was 4.28 (4.92/1.15) for NLE-CDDP and 2.70 (3.21/1.19) for CDDP when SERs were compared between in vivo and jejunal crypt cell experiments.

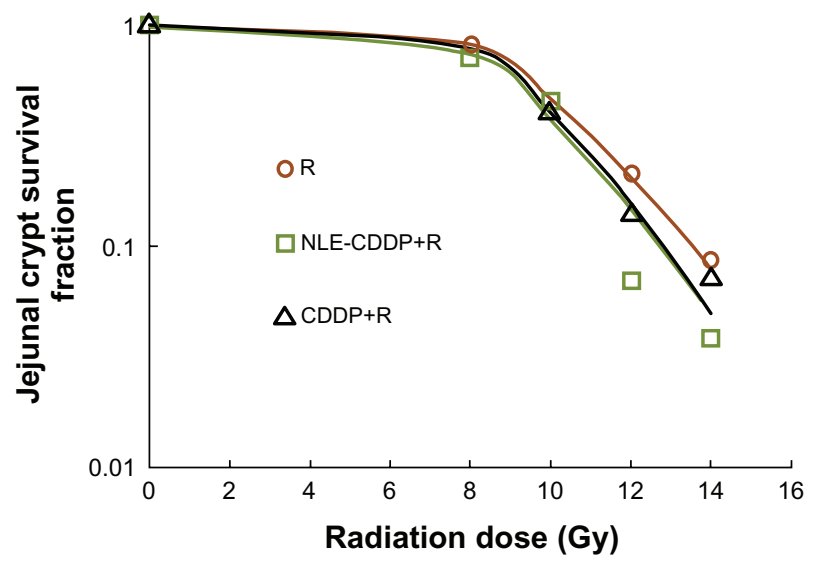

Figure 6 Jejunal crypt cell survivals after irradiation (R), combinations of CDDP and irradiation (CDDP + R), or NLE-CDDP and irradiation (NLE-CDDP $+R$ ) with $72 \mathrm{~h}$ interval. L-Q model: Irradiation alone: $y=\exp \left(0.18 * d-0.026 * d^{2}\right), R^{2}=1.000$ NLECDDP + irradiation: $y=\exp \left(0.202 * d-0.03 * \mathrm{~d}^{2}\right), R^{2}=0.980 \mathrm{CDDP}+$ irradiation: $y=\exp \left(0.218 * d-0.031 * d^{2}\right), R^{2}=0.999$.

Abbreviations: CDDP, cis-platinum diammine dichloride; NLE-CDDP, nanoliposome encapsulated cisplatin; $R$, irradiation-alone. 


\section{Discussion}

Nanoliposomes have been widely studied in cancer treatments as a vector to deliver chemicals into tumor cells, but clinically evident results were limited. The aim of the current study was to synthesize a new NLE-CDDP that could yield a higher tumor concentration and extended CDDP release, and to study its use in combination with radiation as a radiosensitizer.

Burger et al reported that NLE-CDDP could enhance the cytotoxicity and significantly increase cell death up to 1000 times, compared with the same concentration of CDDP. ${ }^{10}$ In our study, IC50 of CDDP against A549 cells was 2.35 times that of NLE-CDDP $(2.63 \mu \mathrm{g} / \mathrm{mL}$ vs $1.12 \mu \mathrm{g} / \mathrm{mL}$ ), which was higher than the 1.34 times reported by Carvalho et al. ${ }^{11}$ In addition, in vivo tests also demonstrated much stronger tumor inhibitions in NLE-CDDP than those in CDDP, indicating that the cytotoxicity of our NLECDDP was more significant than the cytotoxicity of CDDP. The strong toxicity of NLE-CDDP may be attributed to its small size $(100 \mathrm{~nm})$, which is smaller than that reported in the literature $(174 \mathrm{~nm}) .{ }^{11}$ Nanoliposome encapsulated CDDP in liposomes and prevented CDDP from immediately binding with proteins, then CDDP was gradually released directly to the tumor cells through adsorption, lipid exchange, endocytosis, and fusion, by which cell killing was increased. ${ }^{12}$ Ramachandran et al also found that the uptake of nanoparticle formations of CDDP by cells was much easier. ${ }^{13}$

CDDP is a radiation sensitizer and has been widely used with radiation therapy for cancer treatment. ${ }^{14}$ The mechanism of CDDP enhancement of irradiation effect had been studied by $\mathrm{Lu}$ and Kalantari et al, who revealed that the cytotoxicity was enhanced by low-dose cisplatin combined with radiotherapy. The underlying mechanism was the electron-transfer reaction of cisplatin with electrons generated in ionizing irradiation. ${ }^{15,16}$ This finding was similar to what was found in hypoxic cell radiosensitizers, an electron affinity agent. In Lu's study, a low dose of CDDP $(50 \mu \mathrm{M})$ increased irradiation-induced single and double DNA strain breaks, resulting in more cell killing.

In clinical trials, a daily dose of CDDP before radiotherapy was thought to be the best way to improve the efficacy of radiotherapy, but it also caused the long duration of gastrointestinal tract symptoms. ${ }^{17}$ Therefore, although daily low-dose CDDP has a proven radiosensitization effect, its routine use in practice is limited because of its toxicities and inconvenience of administration via intravenous infusion.
Our NLE-CDDP developed in this study was prepared by reverse phase evaporation with a new formulation of sustained release CDDP (100 nm in size, modified by polyethylene glycol on the surface), resulting in slow-release, passive targeting, and long-cycle characteristics. The lipid compositions of the liposome were nontoxic in vitro toxicity tests, suggesting that the liposomes could be potentially used in animals or humans safely.

Our in vitro study demonstrated that both CDDP and NLE-CDDP possessed radiosensitization properties. This was shown by smaller shoulder and slope of cell survival curves in CDDP/NLE-CDDP compared with those of irradiation alone. However, NLE-CDDP has a stronger radiosensitization effect than CDDP (SER of 1.40 vs 1.14 ). Our in vivo study of combination treatments confirmed that the interval between the delivery of NLE-CDDP and irradiation impacted tumor inhibition, with the strongest effect in $72 \mathrm{~h}$ intervals. Thus, in our in vivo radiosensitization study, we used a $72 \mathrm{~h}$ interval.

The in vivo study demonstrated that both CDDP and NLE-CDDP possessed radiosensitization with an SER of 3.21 for CDDP and 4.92 for NLE-CDDP, indicating that NLE-CDDP had stronger radiosensitization. The stronger radiosensitization of NLE-CDDP was probably the benefit of its features of in vivo controlled release and passive targeting, which was consistent with the outcome in a slowrelease formulation of CDDP combined with radiation. ${ }^{18,19}$ Our pharmacokinetic study also demonstrated that free platinum in serum could remain at a concentration of $1.04 \mu \mathrm{g} / \mathrm{mL} 72 \mathrm{~h}$ after injection of NLE-CDDP $(6 \mathrm{mg} / \mathrm{kg})$ through the tail vein, but it could not be detected only $2 \mathrm{~h}$ after CDDP administration, which suggests that NLECDDP could extend in vivo circulation time and maintain a certain concentration for a long time. ${ }^{20}$ In addition, our previous study also confirmed the nanoparticle could aggregate in tumors passively, resulting in concentration of NLE-CDDP in tumors higher than that of CDDP. ${ }^{20}$ Due to its release function, our NLE-CDDP could be accumulated in tumors and released slowly and continuously, like a drug delivery pump, thereby keeping the tumor exposed to drug continuously. When tumors were irradiated, the drugs deposited in tumors played the role of radiosensitizer. In contrast, injected CDDP was quickly bound with proteins and excreted from the body, maintaining a low concentration in tumor for a short time. This elucidates the potential underlying mechanism for the stronger radiosensitization of NLE-CDDP than of CDDP. 
It has been reported that CDDP increased irradiation injury. ${ }^{21}$ We used jejunal crypt cell assay to investigate whether our NLE-CDDP would increase toxicity. When combined with irradiation, NLE-CDDP yielded lower survival of crypt cells than did irradiation alone, with SER of 1.15, whereas CDDP also sensitized irradiation injury of jejunal crypt cell with SER of 1.19. Nevertheless, NLE-CDDP did not show significant increased intestinal injury compared with CDDP. The explanation for less radiosensitization of NLE-CDDP for jejunal crypt cell than for tumors was due to the difference in drug distribution. NLE-CDDP could accumulate in tumors much easier than in normal tissues due to the enhanced permeability and retention (EPR) effect. EPR effect is a property by which certain sizes of molecules, typically liposomes or macromolecular drugs, tend to accumulate in tumor tissue much more than they do in normal tissues. ${ }^{22-24}$ In general, tumor vessels are usually abnormal in form and architecture. They are poorly aligned defective endothelial cells with wide fenestrations, lacking a smooth muscle layer or innervation with a wider lumen, and with impaired functional receptors for angiotensin II. All those factors will lead to abnormal molecular and fluid transport dynamics, especially for macromolecular drugs. The EPR effect helps to carry the nanoparticles and spread them in tumors. However, there is no EPR effect for normal tissue because of their normal vessels.

TGF was 4.28 for NLE-CDDP and 2.70 for CDDP in both SERs for Lewis lung carcinoma (in vivo) and jejunal crypt cell. Therefore, the NLE-CDDP developed in the current study showed its potential as a radiosensitizer in irradiation therapy and warrants further investigation.

In summary, NLE-CDDP could inhibit A549 cells in vitro with an IC50 of $1.12 \mu \mathrm{g} / \mathrm{mL}$, and its toxicity was 2.35 times that of CDDP. When combined with irradiation, an in vitro study of A549 cell line confirmed its radiosensitization with SER of 1.40. An in vivo study showed that the strongest radiosensitization was found in the $72 \mathrm{~h}$ interval between NLE-CDDP and irradiation. When used $72 \mathrm{~h}$ prior to irradiation in Lewis lung carcinoma-bearing mice, NLE-CDDP yielded SER of 4.92. In both in vitro and in vivo studies, SERs were higher for NLE-CDDP than those for CDDP. NLE-CDDP also enhanced radiation injury to jejunal crypt cells with SER of 1.15, but TGF was 4.28.

\section{Acknowledgments}

This study was sponsored by a grant from the Shanghai Science and Technology Committee (nr 0552nm44).
The authors thank Dr Jiade J Lu for his assistance in editing this article.

\section{Disclosure}

No conflicts of interest were declared in relation to this paper.

\section{References}

1. Stathopoulos GP, Boulikas T, Vougiouka M, Rigatosi SK, Stathopoulos JG. Liposomal CDDP combined with gemcitabine in pretreated advanced pancreatic cancer patients: A phase I-II study. Oncol Rep. 2006;15:1201-1204.

2. Xiao C, Qi XR, Maitani Y, Nagai T. Sustained release of CDDP from multivesicular liposomes: potentiation of antitumor efficacy against S180 murine carcinoma. J Pharma Sci. 2004;93(7):1149-1157.

3. Burger KNJ, Staffhorst RWHM, Vijlder HECD, et al. Nanocapsules: lipid-coated aggregates of CDDP with high cytotoxicity. Nat Med. 2002;8(1):81-84.

4. Ramachandran S, Quiwt AP, Kumar S, Lal R. CDDP nanoliposomes for cancer therapy: AFM and fluorescence imaging of CDDP encapsulation, stability, cellular uptake, and toxicity. Langmuir. 2006;22: 8156-8162.

5. Han I, Kim OJ, Lee GY, Sung YK, Song R, Sohn Y. Enhanced antitumor activity of trans (1,2-Diaminocyclohexaneglutamatoplatinum (II)) formulated with stealth liposome. Bioorg Med Chem. 2003;11: 5443-5447.

6. Rosenthal DI, Yom SS, Liu L, et al. A Phase I study of SPI-077 (Stealth liposomal CDDP) concurrent with radiation therapy for locally advanced head and neck cancer. Invest New Drugs. 2002;20:343-349.

7. Koukourakis MI, Coukouraki S, Giatromanolaki A, et al. Liposomal doxorubicin and conventionally fractionated radiotherapy in the treatment of locally advanced non-small-cell lung cancer and head and neck cancer. J Clin Oncol. 1999;17:3512-3521.

8. Huang SK, Lee KD, Hong K, Friend DS, Papahadjopoulos D. Microscopic localization of sterically stabilized liposomes in colon carcinoma-bearing mice. Cancer Res. 1992;52:5135-5143.

9. Szoka F, Papahadjopoulos D. Procedure for preparation of liposomes with large internal aqueous space and high capture by reverse-phase evaporation. Proc Natl Acad Sci. 1978;75(9):4194-4198.

10. Burger KN, Staffhorst RW, Velinova MJ, et al. Nanocapsules: lipid-coated aggregates of CDDP with high cytotoxicity. Nat Med. 2002;8(1):81-84.

11. Carvalho Júnior AD, Vieira FP, de Melo VJ, et al. Preparation and cytotoxicity of CDDP-containing liposomes. Braz J Med Biol Res. 2007;40(8):1149-1157.

12. Jia W, Gao WY. New Formulations of Drug Controlled Release. Beijing: Chemical Industry Press; 2005:313.

13. Ramachandran S, Quist AP, Kumar S, et al. CDDP nanoliposomes for cancer therapy: AFM and fluorescence imaging of CDDP encapsulation, stability, cellular uptake, and toxicity. Langmuir. 2006; 22(19): 8156-8162.

14. Joschko MA, Webster LK, Bishop JF, et al. Radioenhancement by CDDP with accelerated fractionated radiotherapy in a human tumour xenograft. Cancer Chemother Pharmacol. 1997;40(6):534-539.

15. Lu QB. Molecular reaction mechanisms of combination treatment of low-dose cisplatin with radiotherapy and photodynamic therapy. $J \mathrm{Med}$ Chem. 2007;50:2601-2604.

16. Lu QB, Kalantari S, Wang CR. Electron transfer reaction mechanism of cisplatin with DNA at the molecular level. Mol Pharmaceutics. 2007; 4(4):624-628.

17. Schaake-Koning C, van den Bogaert W, Dalesio O, et al. Effects of concomitant CDDP and radiotherapy on inoperable non-small cell lung cancer. N Engl J Med. 1992;326:524-530. 
18. Harrington KJ, Rowlinson-Busza G, Syrigos KN, et al. Pegylated liposome-encapsulated doxorubicin and CDDP enhance the effect of radiotherapy in a tumor xennograft model. Clin Cancer Res. 2000;6: 4939-4949.

19. Ning S, Yu N, Brown DM, Kanekal S, Knox SJ. Radiosensitization by intratumoral administration of CDDP in a sustained-release drug delivery system. Radiother Oncol. 1999;50:215-223.

20. Zhang XM, Ge YQ, Jiang GL, et al. Pharmacokinetics of CDDP packaged in a nanometer liposome. China Oncol. 2007;17(11): $715-718$.

21. Douple EB, Eaton WL, Tulloh ME. Skin radiosensitization studies using combined cis-dichlorodiammineplatinum (II) and radiation. Int J Radiat Oncol Biol Phys. 1979;5(8):1383-1385.
22. Matsumura $Y$, Maeda $H$. A new concept for macromolecular therapeutics in cancer chemotherapy: mechanism of tumoritropic accumulation of proteins and the antitumor agent smancs. Cancer Res. 1986;46(12 Pt 1): 6387-6392.

23. Maeda H, Wu J, Sawa T, Matsumura Y, Hori K. Tumor vascular permeability and the EPR effect in macromolecular therapeutics: a review. $J$ Control Release. 2000;65(1-2):271-284.

24. Vasey PA, Kaye SB, Morrison R, et al. Phase I clinical and pharmacokinetic study of PK1 [N-(2-hydroxypropyl) methacrylamide copolymer doxorubicin]: first member of a new class of chemotherapeutic agents-drug-polymer conjugates. Cancer Research Campaign Phase I/II Committee. Clin Cancer Res.1999;5(1):83-94.

\section{Publish your work in this journal}

The International Journal of Nanomedicine is an international, peerreviewed journal focusing on the application of nanotechnology in diagnostics, therapeutics, and drug delivery systems throughout the biomedical field. This journal is indexed on PubMed Central, MedLine, CAS, SciSearch $\AA$, Current Contents ${ }^{\circledR} /$ Clinical Medicine,
Journal Citation Reports/Science Edition, EMBase, Scopus and the Elsevier Bibliographic databases. The manuscript management system is completely online and includes a very quick and fair peer-review system, which is all easy to use. Visit http://www.dovepress.com/ testimonials.php to read real quotes from published authors. 